\title{
Selection of fiber orientation around geometric discontinuities in designing against failure of GFRP laminates
}

\author{
Roselita Fragoudakis ${ }^{1 *}$ \\ ${ }^{1}$ Merrimack College, Department of Mechanical Engineering, North Andover, USA
}

\begin{abstract}
Determining fiber orientation around geometric discontinuities is challenging and simultaneously crucial when designing laminates against failure. This study presents an approach for selecting the fiber orientations in the vicinity of a geometric discontinuity. Maximum stresses in the discontinuity region are calculated using Classical Lamination Theory and the stress concentration factor. The use of the Tsai-Hill and Tsai-Wu failure theories estimate the minimum moment to cause failure in a lamina. Fiber orientations around the discontinuity are obtained using the Tsa-Hill failure theory.
\end{abstract}

\section{Introduction}

Fiber reinforced laminates have become optimum material alternatives to metals in major applications and industries. Examples are fiber reinforced laminated leaf springs in vehicle suspension systems. This type of composites of high strength to weight ratio and the flexibility to select and optimize the reinforcing phase (fibers) orientation allow for the desired stiffness, mechanical properties, and performance of the structure. Research has shown that replacement of monoleaf steel springs with a laminated Glass Fiber Reinforced (GFRP) leaf, significantly reduces the weight of the leaf spring by more than $20 \%$, without sacrificing strength and performance [1].

Fibers hold the load carrying capacity of Fiber Reinforced Plastics (FRP). Long unidirectional fibers offer high strength and stiffness to laminated FRP beams. However, geometric discontinuities, such as holes, fillets, and tapered edges interrupt the unidirectionality of the fibers and may affect the properties and performance of the laminate. Similar to metal beam structures, a high stress concentration area is observed around the discontinuity region. A simple FE analysis shows a $40 \%$ increase in the stresses developed around a $0.6 \mathrm{~mm}$ hole in a laminate of stacking sequence $\left[0^{\circ}\right]_{3}$.

Composite laminate failure may be due to microscopic failure through fracture of individual material constituents (i.e. fibers and/or matrix), debonding at the consitutents interface, or macroscopic delamination. As a result, during manufacturing of the geometric discontinuity catastrophic failure may occur due to any of the above failure modes.

Failure criteria and models help in the prediction of failure and evaluation of critical stresses. Different criteria predict different failure modes. For example Hashin's [2] theory focuses on fiber failure, while newer approaches can predict fiber and interfiber failure [3]. The Waddoups- Eisenmann-Kaminski (WEK) model and its modifications discussed in literature [4-5] use the stress intensity factors to evaluate the strength of notched composite specimens. However, evaluating failure is one aspect of composite laminate performance, while designing against failure is another.

\footnotetext{
*Corresponding author: fragoudakisr@merrimack.edu
} 
Choosing an optimum stacking sequence can improve the onset of first-ply failure in GFRP laminates. Previous research has shown that symmetric general stacking $\left([0 / 45]_{\mathrm{s}}\right)$ laminates can withstand higher loads before first ply failure occurs, compared to other stacking sequence configuratuions such as cross-ply ([90/0 $]_{\mathrm{s}}$ ) or $[60 / 90 / 45]_{\mathrm{s}}$ (Table 1) [6]. Consequently, choosing the appropriate stacking sequence at the discontinuity vicinity is of equal importance.

Because the fibers cannot be interrupted by the discontinuity, in order to strengthen the vicinity of the discontinuity, different fiber orientations should be selected for this region. Selection of fiber orientation in this region is a challenging process, and depends on a variety of parameters. Different methods have been presented in literature, in an effort to design algorithms to determine such orientations. Goteti and Reddy used Classical Lamination Theory (CLT) and the stress intensity around a circular hole, to examine the effect of fiber angle, hole size, and volume fraction on the stress concentration around the hole [7]. Sharma determined the stress concentration around circular/elliptical/triangular cutouts using Muskhelishvili's complex variable method and fiber orientation as inputs [7]. An effort to fully determine the fiber orientation in a broader domain around the discontinuity, while keeping the lamina fiber orientation unidirectional far from the prescribed domain around the discontinuity, is given by Huang and Haftka [9]. This research uses the Tsai-Wu interactive failure theory to determine the fiber orientation around the discontinuity that will not cause lamina failure. All of the above work agrees that the parameters affecting fiber orientation around discontinuities, such as holes, depend on the size of the discontinuity, the load type and direction, as well as volume fraction of the fibers, which is widely known to affect the mechanical properties of the fiber/epoxy material.

This paper discusses a slightly different approach to determine fiber orientation around circular discontinuities. The loading condition examined is not axial loading as in the aforementioned research, but rather three point bending. The approach focuses on using CLT in conjuction with the Tsai-Hill and Tsai-Wu failure theories to determine the minimum moment to cause failure in the laminate in the absence of a discontinuity, and the effect of the geometric stress concentration factor under bending, to determine the momen to cause failure in the presence of a circular hole. The fiber oreintation in the viccinity of the hole is determined based on this latter minimum moment.

\section{Optimizing Fiber Orientation around a Circular Hole}

\subsection{Laminate Beam}

CLT determines the stress distribution in a semi-infinite beam. As a result, it is important to determine the number of lamina in the laminate, the thickness of each lamina, and the fiber orientation in each lamina.

To determine the minimum moment to cause failure in the laminate, a beam with no discontinuities and unidirectional fibers is considered. The laminate is considered to be symmetric, with a general stacking sequence $\left([0 / 45 / 0]_{s}\right)$ (Fig. 1). A total of 6 layers, each of thickness $1 \mathrm{~mm}$ are considered. The number of layers and their thickness are chosen arbitrarily to facilitate calculations. Typically, GFRP lamina tend to be thinner than $1 \mathrm{~mm}$. The number of layers in the laminate has a significant effect on the stiffness and consequently the stress distribution in the laminate. The beam material is S2 glass fiber/epoxy at 55\% volume fraction. That is a medium to high stiffness GFRP, with properties that are commonly used in the industry. The mechanical properties of the GFRP material are given in Table 1. As can be seen from the properties of the GFRP, the lamina is considered to be transversely isotropic, with direction 1 along the fibers, and the isotropy plane being 23 . 


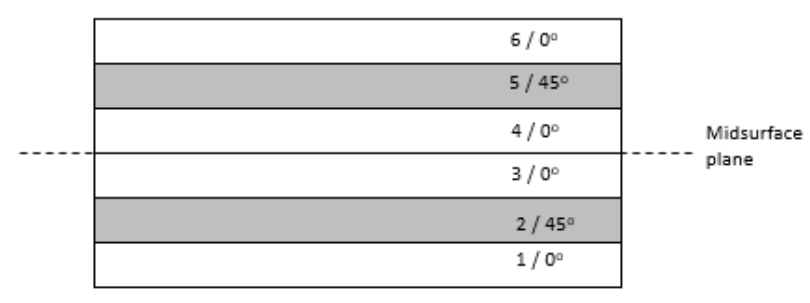

Fig. 1. Stacking Sequence

A beam of the exact same dimensions, stacking sequence, and fiber orientations with a central circular hole of $1 \mathrm{~cm}$ in diameter, is considered in the investigation of the fiber orientations around the discontinuity (Fig. 2).
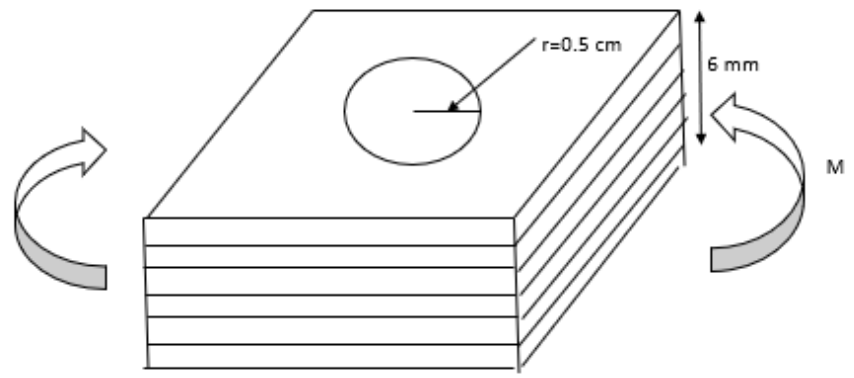

Fig. 2. Laminate with hole

In both of the above cases the loading condition is a bending moment, which is left to be determined using the two failure theories.

Table 1. Properties of S2 glass fiber/epoxy

\begin{tabular}{c|c}
\hline $\mathrm{E}_{1}$ & $34 \mathrm{GPa}$ \\
$\mathrm{E}_{2}$ & $8.9 \mathrm{GPa}$ \\
$\mathrm{G}_{12}$ & $4.5 \mathrm{GPa}$ \\
$\mathrm{v}_{12}$ & 0.27 \\
X (Longitudinal tensile strength) & $2000 \mathrm{MPa}$ \\
$\mathrm{X}^{\prime}$ (Longitudinal compressive strength) & $1240 \mathrm{MPa}$ \\
Y (Transverse tensile strength) & $49 \mathrm{MPa}$ \\
$\mathrm{Y}^{\prime}$ (Transverse compressive strength) & $158 \mathrm{MPa}$ \\
S (Shear strength) & $63 \mathrm{MPa}$ \\
\hline
\end{tabular}

\subsection{The Vicinity around the Hole}

The region around a geometric discontinuity affected by the maximum stress concentration can be approximated to the plastic zone. To determine this zone a very small crack is assumed 
just on the verge of the hole. This crack, in the epoxy matrix of the GFRP, has the potential to propagate and lead to fracture of the matrix, which is one of the failure modes in FRP composites. Assuming a very small crack, means that the ratio of crack length to hole radius approaches zero. Using this ratio, the mode I stress intensity factor $\left(\mathrm{K}_{\mathrm{I}}\right)$ can be calculated using the equation for cracks at the edge of a circular hole (Eq. 1). Assuming that the stress intensity factor becomes equal to the critical stress intensity factor $\left(\mathrm{K}_{\mathrm{c}}\right)$ when the crack begins to propagate and cause failure, the radius of the plastic zone ( $\mathrm{r}_{\mathrm{y}}$ ) can be estimated (Eq. 2 )

$$
\begin{gathered}
K_{I}=\sigma \sqrt{\pi \alpha} f(\alpha / r) \\
r_{y}=\frac{1}{2 \pi}\left(\frac{K_{c}}{\sigma_{y}}\right)^{2}
\end{gathered}
$$

where $\alpha$ is the crack length, $r$ the hole radius, and $\sigma_{\mathrm{y}}$ the applied yield stress.

The radius of the plastic zone begins at the crack tip. Assuming that the crack length is very small, this radius can be assumed to begin at the rim of the circular hole and determine the extent of the crucial vicinity around the discontinuity, where a different reinforcing stacking sequence should be selected. The above equations clearly show the effect of the hole size, to the selection of this zone.

\subsection{CLT, failure theories, and maximum stress}

CLT uses the information of the material properties and fiber orientation in each lamina to generate a stiffness matrix $(\bar{Q})$ for every lamina (Eq. 3). The position of each lamina in the matrix (z), the lamina thickness ( $\mathrm{t}$ ), the loading of the laminate ( $\mathrm{F}$ and/or $\mathrm{M}$ - in the case of this paper only a bending moment along the longitudinal direction of the beam is considered), build the stress distribution in the $\mathrm{k}^{\text {th }}$ lamina (Eq. 4).

$$
\begin{gathered}
\bar{Q}_{k}=\left[\begin{array}{lll}
\bar{Q}_{11} & \bar{Q}_{12} & \bar{Q}_{16} \\
\bar{Q}_{21} & \bar{Q}_{22} & \bar{Q}_{26} \\
\bar{Q}_{61} & \bar{Q}_{62} & \bar{Q}_{66}
\end{array}\right] \\
\left\{\begin{array}{c}
\sigma_{x} \\
\sigma_{y} \\
\tau_{x y}
\end{array}\right\}_{k}=\left[\bar{Q}_{k}\right]\left(\left\{\begin{array}{c}
\varepsilon_{x}^{o} \\
\varepsilon_{y}^{o} \\
\gamma_{x y}^{o}
\end{array}\right\}+z\left\{\begin{array}{c}
\kappa_{x} \\
\kappa_{y} \\
\kappa_{x y}
\end{array}\right\}-\left\{\begin{array}{c}
\alpha_{x} \\
\alpha_{y} \\
\alpha_{x y}
\end{array}\right\}_{k} \Delta T\right)
\end{gathered}
$$

where $\varepsilon_{\mathrm{ij}}^{\mathrm{o}}$ are the mid-surface strains developed on the midsurface plane separating the laminate into two equal top and bottom portions (Fig. 1), $\kappa_{\mathrm{ij}}$ are the laminate curvatures, and $\alpha_{\mathrm{ij}}$ the coefficient of thermal expansion which accounts for the thermal strains developed in the laminate. Such strains may occur during manufacturing when $\Delta \mathrm{T}$ becomes significantly large and residual strains between layers may be induced. Thermal effects are ignored in the current study.

The above stress distribution is evaluated at global axis $(\mathrm{x}, \mathrm{y})$ which remain the same for all laminae. However, because CLT focuses on plane analysis, and failure theories account for failure within a single lamina, the above stresses are translated to principal stresses, along the local axis of each lamina $(1,2)$. The failure theories considered in this study are interactive failure theories, i.e. account for the interaction of stresses in different directions. To determine the minimum moment to cause failure in each ply the Tsai-Hill (Eq. 5) and $\mathrm{Tsai}-\mathrm{Wu}$ (Eq. 6) failure theories are considered. 


$$
\begin{gathered}
\frac{\sigma_{1}^{2}}{X^{2}}-\frac{\sigma_{1} \sigma_{2}}{X^{2}}+\frac{\sigma_{2}^{2}}{Y^{2}}+\frac{\tau_{12}{ }^{2}}{S^{2}} \leq 1 \\
F_{11} \sigma_{1}{ }^{2}+2 F_{11} \sigma_{1} \sigma_{2}+F_{22} \sigma_{2}{ }^{2}+F_{66} \sigma_{6}{ }^{2}+F_{1} \sigma_{1}+F_{2} \sigma_{2} \leq 1
\end{gathered}
$$

where the coefficients in the Tsai-Wu failure theory depend on the strengths of the material.

The stresses calculated in Eq. 4 are the stresses of a single lamina, of unidirectional fibers, and no geometric discontinuities. To account for these discontinuities the geometric stress concentration factor $\left(\mathrm{K}_{\mathrm{t}}\right)$ is used to multiply these stresses and maximize them to account for the effect of the hole. For the aforementioned dimensions of the laminate and hole $\mathrm{K}_{\mathrm{t}}=2.7$. However, this factor changes depending on the size of the hole and dimensions of the laminate. This is another area where we see the effects of the hole size not just to the stresses in laminate, but also to the failure of each lamina.

\subsection{The optimization process}

Using the equations and with the constraints mentioned above, the smallest moments to cause failure in each ply are shown in Table 2 . The smallest of these moments is applied to the plate with the central circular hole.

Table 2 shows the absolute values of the minimum moments. Tsai-Hill, with the minimum condition to cause failure when Eq. 5 is equal to 1, gives two possible moments that can cause failure. They both have the same magnitude, while the set of two moments accounts for clockwise and counterclockwise direction. On the other hand, for the same failure condition, the Tsai-Wu theory gives a positive and negative moment at each layer, however their magnitudes are not the same. Table 2 shows the moment with the smaller magnitude. Either failure theory, predicts that the $0^{\circ}$ orientation is the strongest, and the symmetry of the matrix allows for a mirror image of the moments above and below the midsurface plane. A final conclusion of this analysis, is that Tsai-Wu tends to overestimate failure, the lamina fail at lower loads, while Tsai-Hill tends to underestimate failure. Since failure theories are used only to approximate failure, without physical experiments it is not easy to choose one over the other.

Assuming that the discontinuity is at the center of a semi-infinite plate, the lamina can be treated as symmetric in both the $\mathrm{x}$ and $\mathrm{y}$ directions of its plane. To choose the number of points required around the hole, in order to determine the path of the fiber orientation, the finite element concept of appropriate seeds for geometric discontinuities is applied. A minimum of 16 seeds around a circle is recommended, as a result a total of 4 seeds in a quadrant. Following Huang and Haftka's [9] model of fiber orientation, outside the plastic zone the fiber orientation returns to that of the lamina, as prescribed from the general laminate stacking sequence. The number of seeds inside the plastic zone depends on the accuracy of the results and radius of the plastic zone. In this paper, the optimization for the fiber orientation around the hole is constrained to the first 4 seeds. The possible orientations for theses seeds can vary between $-90^{\circ}$ and $90^{\circ}$. 
Table 2. Absolute values of minimum moment to cause lamina failure

\section{Lamina / Fiber \\ Orientation}

Moment $[\mathbf{N m} / \mathbf{m}]$

Tsai-Hill=1
Moment $[\mathbf{N m} / \mathbf{m}]$

\author{
Tsai-Wu=1
}

$1 / 0^{\circ}$

$2 / 4^{\circ}$

$3 / 0^{\circ}$

$4 / 0^{\circ}$

$5 / 45^{\circ}$

$6 / 0^{\circ}$
269

72

808

808

72
67

512

512

67

171

171

The failure theories, as applied above, assume a load carried along the direction of the fibers. However, in the vicinity of the discontinuity the fibers will be constantly changing orientation (along the new four orientations). To account for this effect on the load, the minimum load factor is calculated as the positive root of a polynomial constructed base on the Tsai-Hill failure theory at the instance it is equal to 1 (Eq. 7).

$$
\left(\frac{\sigma_{1}^{2}}{X^{2}}-\frac{\sigma_{1} \sigma_{2}}{X^{2}}+\frac{\sigma_{2}^{2}}{Y^{2}}+\frac{\tau_{12}{ }^{2}}{S^{2}}\right) \rho^{2}-1=0
$$

The positive load factor $\rho$ is a function of the orientations around the discontinuity.

\section{Results}

The positive roots of Eq. 7 are examined in order to determine the appropriate orientations around the vicinity of the discontinuity. The orientations are estimated for the case that the positive load factor becomes 1, and Eq. 7 becomes Eq. 5 at the first possible occurrence of failure. A similar approach can be followed using the Tsai-Wu criterion. Huang and Haftka's follow this approach for the case of axial loading [9].

Table 3 shows possible orientations for the four angles around the discontinuity. It can be observed that there is a gap in the calculation of these orientations between $40^{\circ}$ and $71^{\circ}$. Solutions indicate a very large range of orientations within these two bounds. To narrow this 
range a genetic algorithm approach can be followed. However, this approach is outside the scope of this paper. Fig. 3 plots orientations in the regions immediately next to the discontinuity, and far away from it, at a radius of $4 \mathrm{~cm}$. This larger radius is calculated as the end of the plastic zone using Eq. 2, for a crack of length $2.5 \mathrm{~mm}$. To prevent the crack tip from coinciding with the fibers, the fiber orientations begin $5 \mathrm{~mm}$ away form the hole edge. This distance of $5 \mathrm{~mm}$ is taken as a safety precaution at twice the length of the crack. However, a more detailed fracture mechanics analysis (also outside the scope of this paper) can help determine this distance with higher accuracy.

Table 3. Possible orientations in the vicinity of the discontinuity at the $0^{\circ}$ orientation lamina

\begin{tabular}{c|c}
\hline & $8^{\circ}$ \\
& $14^{\circ}$ \\
Possible angle values in the vicinity of the discontinuity & $40^{\circ}$ \\
$71^{\circ}$ \\
$72^{\circ}$ \\
$81^{\circ}$ \\
\hline
\end{tabular}
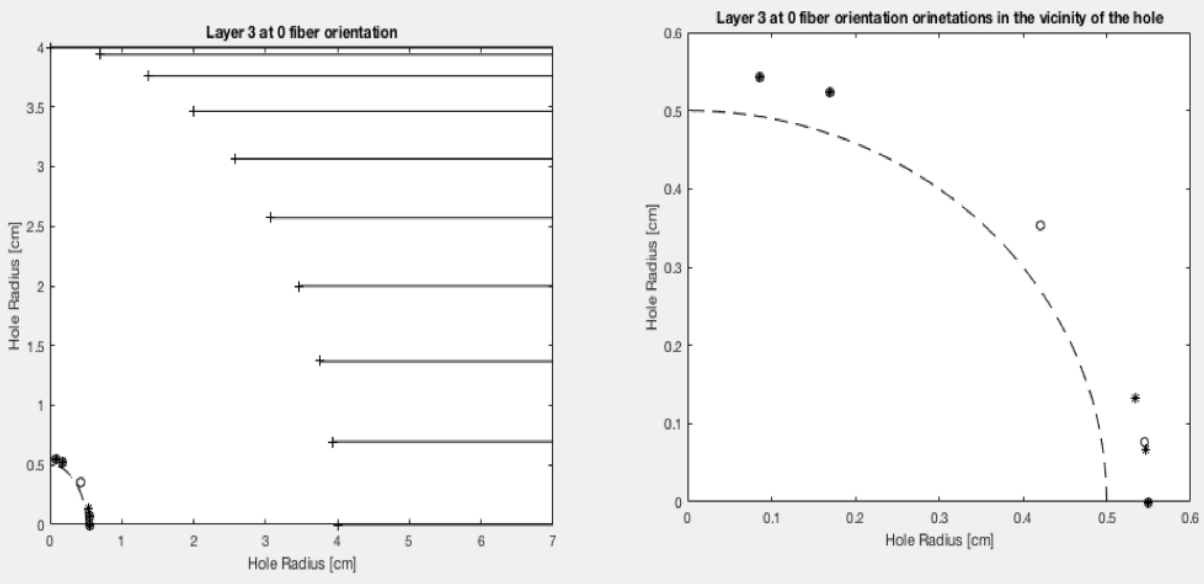

Fig. 3. Fiber orientation around the discontinuity and beyond the plastic zone (left) / Fiber orientation in the vicinity of the discontinuity (right), for Lamina 3 at $0^{\circ}$ fiber orientation

The fiber orientations of Fig. 3 are those for lamina 3, where the general fiber orientation is at $0^{\circ}$. Similar results are obtained for the other laminae $(1,4,6)$ whose general orientation is at $0^{\circ}$. On the other hand, laminae at $45^{\circ}$ appear more challenging in determining these orientations. These cases are not examined in this paper, and results for these layers are expected to be found using a genetic algorithm, which could potentially reduce the wide range of solutions.

\section{Conclusions}

Reinforcing the regions around discontinuities can have a significant effect in the life and performance of a laminate. This paper presents a simple approach to determine a range of possible fiber orientations around a circular hole. The approach depends on the fundamental definitions of stress intensity and stress concentration factors, while CLT and failure theories calculate the stress distribution and failure loading conditions. Analysis is constrained for 
loading under three point bending conditions, which is not typically found in similar research in the literature.

The analysis and results show that for $0^{\circ}$ fiber orientation lamina, the fiber orientations in the vicinity of the discontinuity are not affected by the location of the lamina in the laminate. For the case of $45^{\circ}$ fiber orientation there appear to be a very large range of such orientations for the vicinity of the discontinuity and further optimization is required.

The preliminary analysis of this approach confirms the conclusions of previous research [7-9] that the size and shape of the discontinuity have a significant effect on determining such orientations. Additionally, the dimensions of the laminate and lamina, not only affect the CLT results, but also the effect of the discontinuity in these results.

\section{References}

1. M.M. Shokrieh, D. Razaei, Composite Structure. 60, 317-327, 2003.

2. Z. Hashin, Journal of Applied Mechanics. 47, 329-334, 1980.

3. M.C. Ribeiro, et al., International Journal of Composite Materials, 3, 59-70, 2013.

4. M.E. Waddoups, J.R. Eisenmann, B.E. Kaminski, Journal of Composite Materials, 5, 446-451, 1971.

5. V.K. Kannan, A. Rajadurai, B.N. Nageswara Rao, Journal of Composite Materials, 45, 1031-1043, 2010.

6. R. Fragoudakis, J.A. Gallagher, Procedia Engineering, 200, 221-228, 2017.

7. C. Goteti, S. Reddy, International Journal of Applied Science \& Engineering, 2, 1-12, 2014.

8. D. S. Sharma, Proceedings of the World Congress on Engineering, III, 2011.

9. J. Huang, R. T. Haftka, Structural Multidisc Optimization, 30, 335-341, 2005. 